SANDIA REPORT

SAND96-2148 • UC-406

Unlimited Release

Printed September 1996

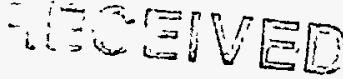

$$
\begin{aligned}
& \text { } 68992 \\
& 08 \pi 1
\end{aligned}
$$

\title{
FIDAP Capabilities for Solving Problems with Stiff Chemistry
}

\author{
J. R. Torczynski, T. A. Baer
}

\section{Prepared by}

Sandia National Laboratories

Albuquerque, New Mexico 87185 and Livermore, California 94550

for the United States Department of Energy

under Contract DE-AC04-94AL85000

Approved for public release; distribution is unlimited.

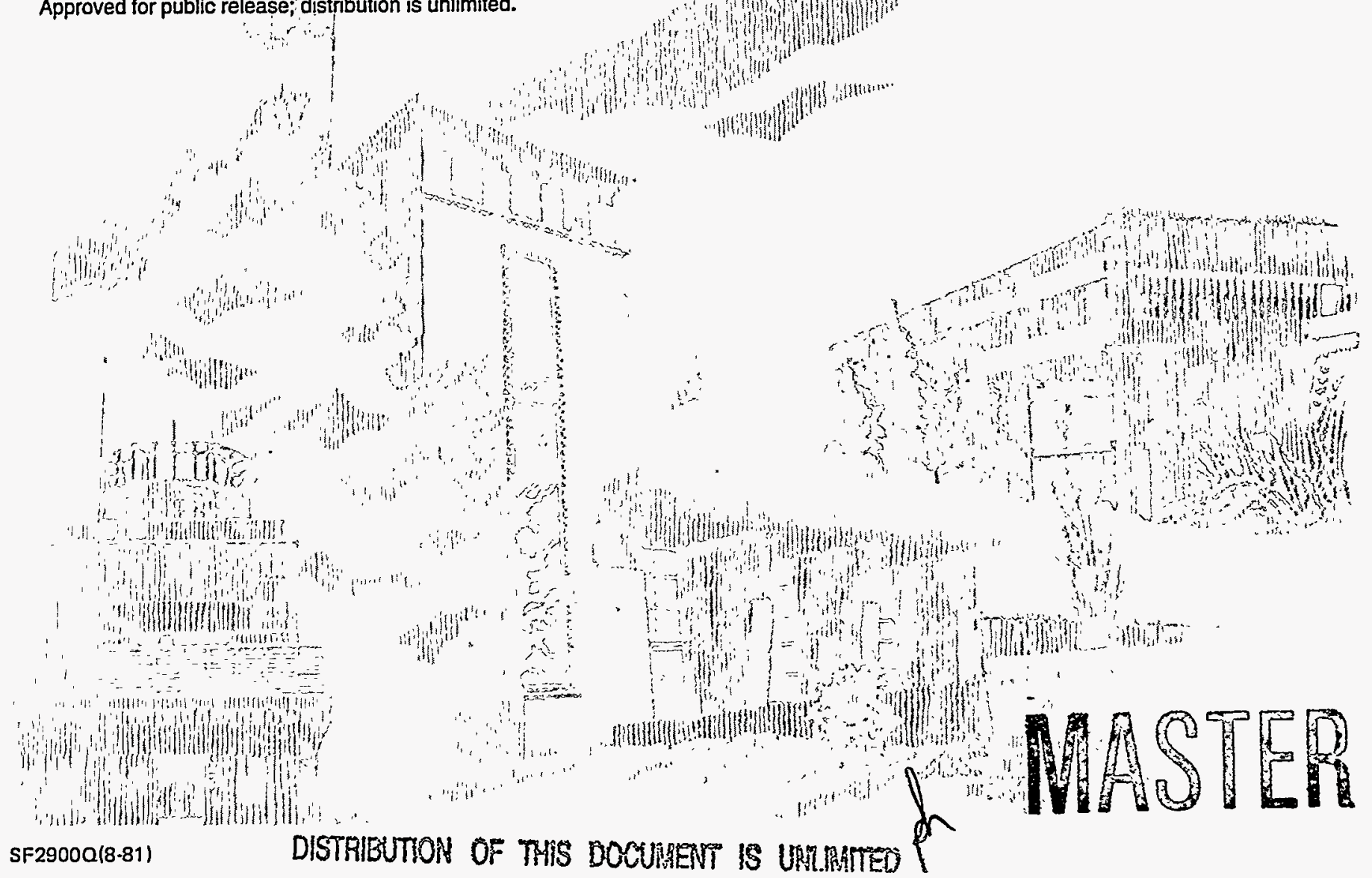


Issued by Sandia National Laboratories, operated for the United States Department of Energy by Sandia Corporation.

NOTICE: This report was prepared as an account of work sponsored by an agency of the United States Government. Neither the United States Government nor any agency thereof, nor any of their employees, nor any of their contractors, subcontractors, or their employees, makes any warranty, express or implied, or assumes any legal liability or responsibility for the accuracy, completeness, or usefulness of any information, apparatus, product, or process disclosed, or represents that its use would not infringe privately owned rights. Reference herein to any specific commercial product, process, or service by trade name, trademark, manufacturer, or otherwise, does not necessarily constitute or imply its endorsement, recommendation, or favoring by the United States Government, any agency thereof or any of their contractors or subcontractors. The views and opinions expressed herein do not necessarily state or reflect those of the United States Government, any agency thereof or any of their contractors.

Printed in the United States of America. This report has been reproduced directly from the best available copy.

Available to DOE and DOE contractors from

Office of Scientific and Technical Information

PO Box 62

Oak Ridge, TN 37831

Prices available from (615) 576-8401, FTS 626-8401

Available to the public from

National Technical Information Service

US Department of Commerce

5285 Port Royal Rd

Springfield, VA 22161

NTIS price codes

Printed copy: A03

Microfiche copy: A01 
SAND96-2148

Unlimited Release

Printed September 1996
Distribution

Category UC-406

\title{
FIDAP Capabilities for Solving Problems with Stiff Chemistry
}

\author{
J. R. Torczynski \\ Energetic and Multiphase Processes Department \\ Sandia National Laboratories \\ Albuquerque, New Mexico 87185 \\ T. A. Baer \\ Gram, Inc. \\ Albuquerque, NM 87112
}

\begin{abstract}
In support of the Motorola CRADA, the capabilities of the computational fluid dynamics code FIDAP (Fluid Dynamics International) for simulating problems involving fluid flow, heat transport, and chemical reactions have been assessed and enhanced as needed for semiconductor-processing applications (e.g. chemical vapor deposition). A novel method of treating surface chemical species that uses only pre-existing FIDAP commands is described and illustrated with test problems. A fullJacobian treatment of the chemical reaction rate expressions during formation of the stiffness matrix has been implemented in FIDAP for both the Arrhenius-parameter and user-subroutine methods of specifying chemical reactions, where the Jacobian terms can be calculated analytically or numerically. This formulation is needed to obtain convergence when reaction rates become large compared to transport rates ("stiff" chemistry). Several test problems are analyzed, and in all cases this approach yields good convergence behavior, even for extremely stiff fluid-phase and surface reactions. A stiff segregated algorithm has been developed and implemented in FIDAP. Analysis of test problems indicates that this algorithm yields improved convergence behavior compared with the original segregated algorithm. This improved behavior enables segregated techniques to be applied to problems with stiff chemistry, as required for large three-dimensional multi-species problems.
\end{abstract}




\section{Acknowledgment}

The authors gratefully acknowledge interactions with Peter Esherick and Harry K. Moffat of Sandia National Laboratories, Michael S. Engelman, Suzanne Caulfield, and Art Gooding of Fluid Dynamics International, and Erik W. Egan of Motorola. 


\section{DISCLAIMER}

Portions of this document may be illegible in electronic image products. Images are produced from the best available original document. 


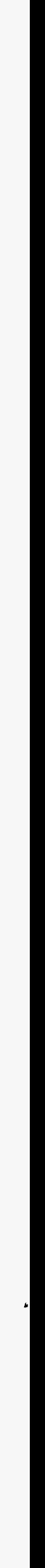




\section{Table of Contents}

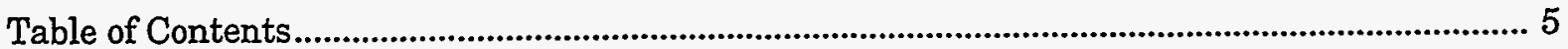

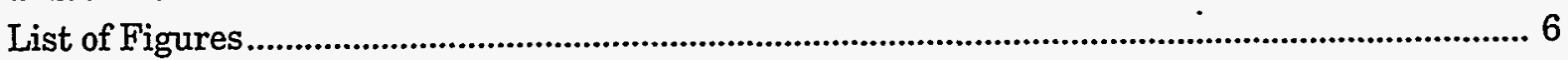

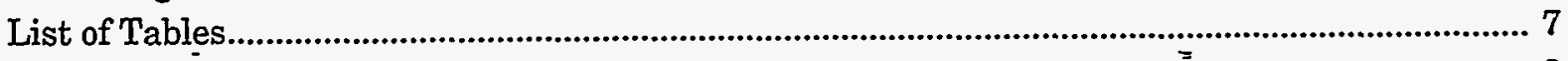

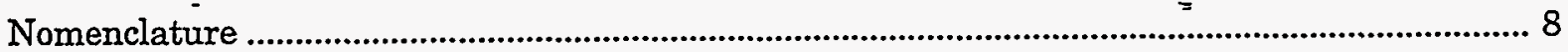

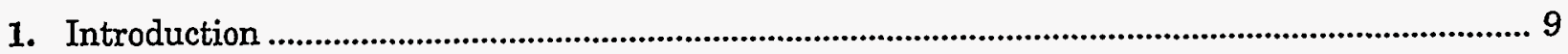

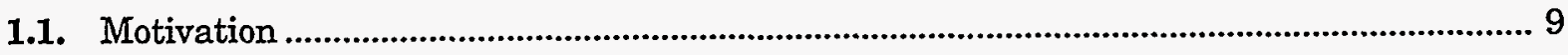

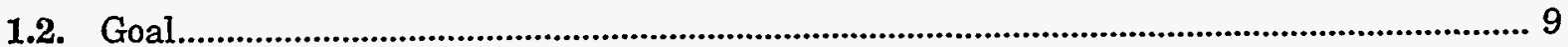

2. Treatment of Surface Chemical Species........................................................................................ 10

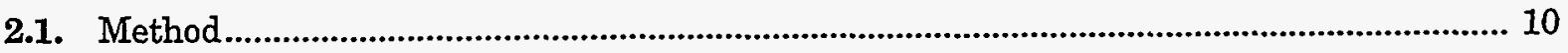

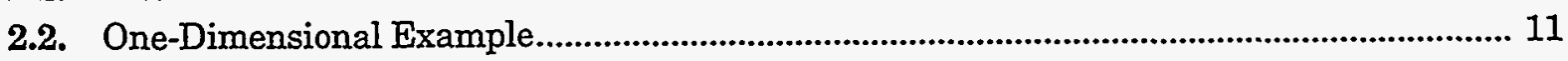

2.3. Two-Dimensional Example ............................................................................................... 13

3. Full-Jacobian Approach for Stiff Chemistry ................................................................................. 15

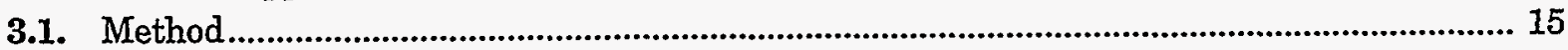

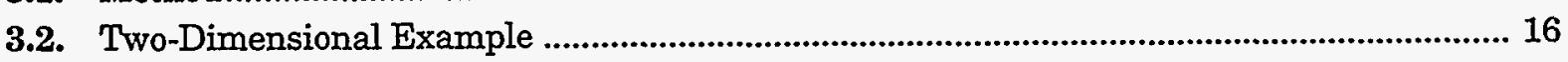

4. Segregated Solution Approach for Stiff Chemistry ....................................................................... 20

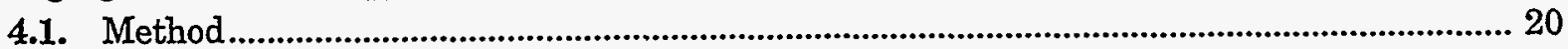

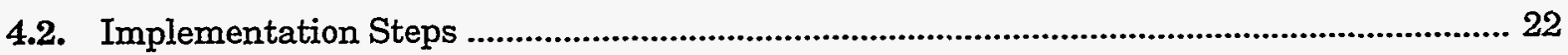

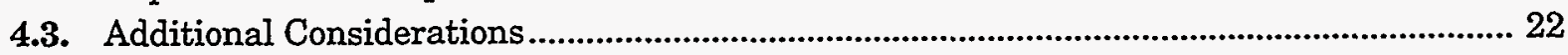

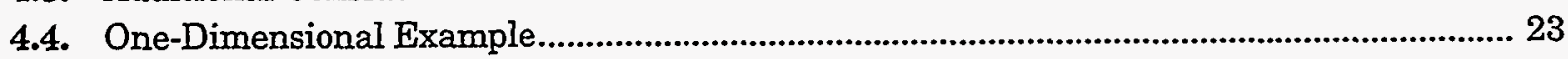

4.5. Three-Dimensional Example................................................................................................ 25

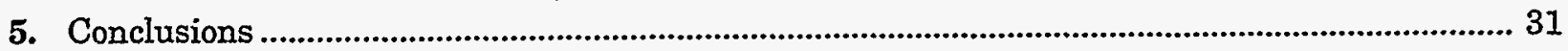

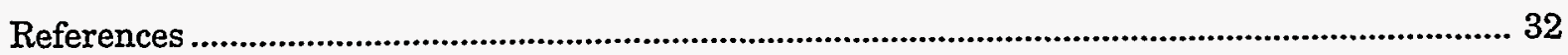

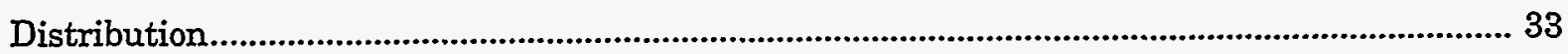




\section{List of Figures}

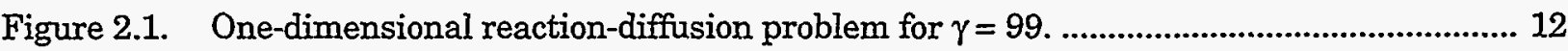

Figure 2.2. Backward-facing step simulation with surface species and reactions. ........................... 14

Figure 3.1. Convergence history for the full-Jacobian backward-facing step simulation. ............... 18

Figure 3.2. Backward-facing step simulation with full-Jacobian approach for $\gamma=10^{3} \ldots \ldots \ldots \ldots \ldots . .19$

Figure 4.1. One-dimensional reaction-diffusion problem with $\gamma=10^{3}$...................................... 24

Figure 4.2. Three-dimensional geometry: top, schematic diagram; bottom, mesh......................... 27

Figure 4.3. Solver dependence of solution along center line across reactive surface for $\gamma=10^{5}$...... 28

Figure 4.4. Convergence behavior of original and stiff segregated solver for $\gamma=10^{5} \ldots \ldots \ldots \ldots \ldots \ldots . . . . .29$

Figure 4.5. Center-line solution behavior of stiff segregated solver prior to convergence............... 30 


\section{List of Tables}

Table 2.1. Information for the surface-species backward-facing step simulations....................... 13

Table 3.1. Information for the full-Jacobian backward-facing step simulations. ......................... 17

Table 4.1. Geometric information for three-dimensional backward-facing step simulation......... 25

Table 4.2. Boundary conditions for three-dimensional backward-facing step simalation. ........... 25

Table 4.3. Material parameters for three-dimensional backward-facing step simulation. ........... 26

Table 4.4. Simulation information for the original and stiff segregated solvers. .......................... 26 


\section{Nomenclature}

\begin{tabular}{|c|c|}
\hline$C$ & specific heat \\
\hline$C^{(m)}$ & capacity of species $m$ \\
\hline$c^{(m)}$ & mass fraction (concentration) of species $m$ \\
\hline$D^{(m n)}$ & matrix containing diagonal portion of $M^{(m n)}$ matrix \\
\hline$E_{1}, E_{2}$ & Arrhenius activation-energy reaction-rate parameters \\
\hline$e$ & element number \\
\hline$H$ & heating-rate parameter \\
\hline$i, j$ & subscript indices denoting nodes or matrix entries \\
\hline$K^{(m n)}$ & species $m$ diffusive-convective stiffness matrix \\
\hline$k$ & thermal conductivity \\
\hline$M^{(m n)}$ & block of Jacobian matrix relating species $m$ and $n$ \\
\hline$\tilde{M}^{(m m)}$ & modified species $m$ stiffness matrix \\
\hline$m, \bar{n}, s$ & superscript indices in parentheses denoting chemical species \\
\hline$N_{s}$ & number of chemical species \\
\hline$N_{n}$ & number of nodes \\
\hline$\hat{n}$ & normal unit vector \\
\hline$p$ & pressure \\
\hline$Q$ & fluid-phase heat-production rate \\
\hline$q$ & surface heat-production rate \\
\hline$R_{i}^{(s m)}$ & matrix used to modify stiffness matrix and right-hand side vector \\
\hline $\operatorname{Re}$ & Reynolds number \\
\hline$S^{(m)}$ & fluid-phase chemical reaction rate of species $m$ \\
\hline$s^{(m)}$ & surface chemical reaction rate of species $m$ \\
\hline$s_{t}$ & total surface chemical reaction rate summed over all species \\
\hline$T$ & temperature \\
\hline$\vec{u}$ & velocity vector \\
\hline$V^{(m)}$ & right-hand side vector for species $m$ \\
\hline$\tilde{V}^{(m)}$ & modified right-hand side vector for species $m$ \\
\hline $\begin{array}{l}x, y, z \\
Z_{i i}^{(n m)} \\
\alpha^{(m)}\end{array}$ & $\begin{array}{l}\text { Cartesian coordinates } \\
\text { matrix inverse of } D_{i i}{ }^{(m n)} \text { matrix over }(m n) \\
\text { diffusivity of species } m\end{array}$ \\
\hline$\beta$ & Arrhenius temperature-dependence reaction-rate parameter \\
\hline$\delta c^{(m)}$ & solution update vector for species $m$ \\
\hline$\delta S^{(m)}$ & chemical-reaction source-term update vector for species $m$ \\
\hline$\partial S^{(m)} / \partial c^{(n)}$ & Jacobian matrix for chemical reactions \\
\hline$\Gamma$ & parameter in closed-form analytical solutions \\
\hline$\gamma$ & stiffness parameter, ratio of reaction rate to transport rate \\
\hline$\rho$ & density \\
\hline$\mu$ & viscosity (absolute) \\
\hline
\end{tabular}




\section{Introduction}

\subsection{Motivation}

In support of the Motorola CRADA, the computational fluid dynamics code FIDAP (Fluid Dynamics International) [1] has been examined for simulating semiconductor-processing applications. The class of problems to be addressed has the following relevant characteristics:

- gas equation of state, significant temperature and density variations

- dynamically incompressible flow, low Mach number

- steady laminar flow, modest Reynolds number

- multiple gas-phase and surface chemical species and reactions

- chemistry often dilute, yielding advection-diffusion situation

- complex geometry, often three-dimensional

FIDAP has been used extensively by Motorola personnel to simulate problems with some of these features [2]. FIDAP solves the Navier-Stokes and energy equations for fluid motion and energy transport. It also treats up to 15 chemical species with mass fractions (concentrations) $c^{(m)}$, which are transported in the fluid and react in the bulk fluid and on reactive surfaces according to

$$
\begin{gathered}
\rho C^{(m)} \vec{u} \cdot \nabla c^{(m)}=\nabla \cdot\left(\rho \alpha^{(m)} \nabla c^{(m)}\right)+S^{(m)}\left(\left\{c^{(n)}\right\}\right) \text { in the fluid, } \\
-\rho \alpha^{(m)} \hat{n} \cdot \nabla c^{(m)}+c^{(m)} s_{t}=s^{(m)}\left(\left\{c^{(n)}\right\}\right), \rho \vec{u} \cdot \hat{n}=s_{t}, s_{t}=\sum_{m} s^{(m)} \text { on the surface. }
\end{gathered}
$$

The fluid-phase and surface chemical-reaction source terms $S^{(m)}$ and $s^{(m)}$ for $c^{(m)}$ can depend on all species concentrations $\left\{c^{(n)}\right\}$. Of particular interest are FIDAP's capabilities in the following areas:

- surface chemical species and reactions

- chemical reactions with rates greatly exceeding transport rates ("stiff" chemistry)

- large problems with chemistry, both three-dimensional and multi-species

\subsection{Goal}

An effort was made to assess FIDAP's capabilities for these types of problems and to enhance FIDAP's capabilities as needed while preserving the FIDAP architecture as much as possible [3]. This report presents the results of this effort. Chapter 2 presents a method of treating surface chemical species (i.e. not existing in the fluid) that uses only pre-existing FIDAP commands, with test problems to illustrate this approach. Chapter 3 presents a method for solving problems when the chemicalreaction rates are large compared to the species-transport rates ("stiff" chemistry) using cross-species information in the stiffness matrix. This method has been implemented in FIDAP for both the Arrhenius-pärameter and user-subroutine methods of specifying chemical reactions, where the required information can be calculated analytically or numerically. Several test problems are presented to illustrate the resulting good convergence behavior, even for extremely stiff fluid-phase and surface reactions. Chapter 4 presents a method for improving the convergence behavior of segregated solution techniques for problems with stiff chemistry and the implementation of this method in FIDAP, along with test problems to demonstrate the improved convergence behavior. 


\section{Treatment of Surface Chemical Species .}

\subsection{Method}

In many semiconductor-processing applications (e.g. chemical vapor deposition), some of the chemical species exist only on reactive surfaces and not within the adjacent fluid phase. Although at first glance it appears to lack the capability to handle surface chemical species, FIDAP can in fact be used to simulate such species in a straightforward fashion. In the remainder of this section, a method for implementing surface chemical species in the context of FIDAP's pre-existing capability to treat fluidphase species is presented. This method involves applying pre-existing FIDAP commands in a novel way and thus requires no custom user subroutines or modifications to FIDAP itself. Examples validating this approach by comparison to analytical solutions are presented.

The following method can be used to treat surface chemical species in FIDAP via FIPREP.

1. Although in reality species $m$ exists only on reactive surfaces and not in the fluid, allow species $m$ to exist in the fluid as an artifice.

2. Define the surface reactions using the SREACTION keyword on the ENTITY card along with the REACTION card.

3. Use the BCNODE card to set the concentration of species $m$ to an arbitrary constant (preferably zero unless a reasonable guess is known) throughout the fluid and on all nonreactive surfaces. The EXCLUSIVE option is used to ensure that the concentration is not set on the reactive surfaces. A FIPREP example is shown below for species 3 .

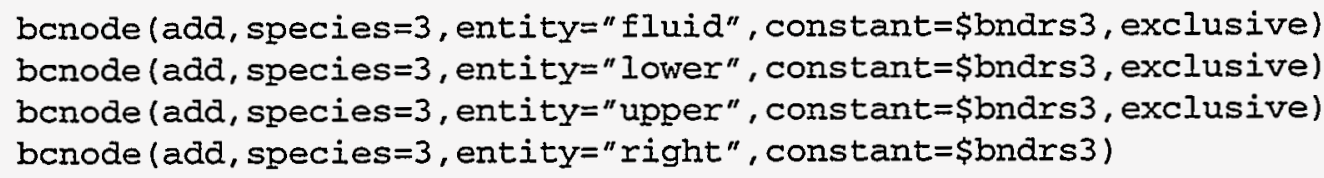

4. Put the SNOCONVECTION=m keyword on the ENTITY card for the fluid to suppress the convective term for species $m$, or (alternatively) set the CAPACITY for species $m$ to a "very small" value (not zero, or FIDAP will default it back to unity) to render the convectivetransport term negligible compared to the chemical-reaction source term. A FIPREP example is shown below for species 3 .

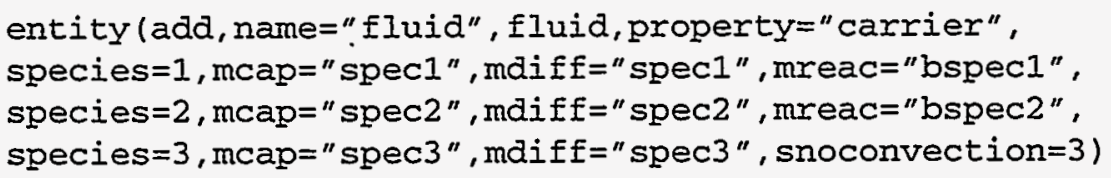

5. Set the DIFFUSIVITY for species $m$ to a "very small" value to render the diffusive fiux negligible compared to the surface-reaction term.

Using this approach, $c^{(m)}$ will be free (a mathematical "unknown") only at nodes on the reactive surfaces. At these nodes, the $c^{(m)}$ flux will be very close to zero: the convective flux is zero since the convection term has been suppressed, and the diffusive flux is negligibly small by virtue of the small diffusivity. Thus, the equation applied at each of these nodes is as desired for a surface species: the surface reaction rate vanishes at steady state, $s^{(m)} \approx 0$. Note that surface diffusion is absent. 
This approach has several advantages.

1. No modifications to the FIDAP source code or custom user subroutines are required.

2. The problem size remains manageable: since the surface species concentrations are "known" (arbitrary constants) away from the reactive surfaces, they do not enter into the matrix equation as additional unknowns.

3. Since the diffusivities of surface species are taken to be small, unphysical lateral diffusive transport of surface species on the reactive surfaces is minimized.

4. Other quantities existing only on the surface such as area density of unoccupied sites for surface reactions can probably be treated in the same manner.

This approach also has some limitations.

1. Although the diffusivities are small, they are nonzero, leading to some transport of surface species away from the surface and into the fiuid. This is mitigated by the small size of the diffusivities.

2. The small size of the diffusivities makes this a numerically stiff problem. This is mitigated by modifying FIDAP to form the full Jacobian of the chemical-reaction source terms, as discussed in subsequent chapters of this report.

3. - It must be recognized that the "mass fraction" variables of surface species should not be interpreted as mass fractions in the fluid phase. The chemical model being employed entirely determines the interpretations assigned to these variables (e.g. area density of unoccupied surface sites).

\subsection{One-Dimensional Example}

As a test case, a one-dimensional diffusion problem with three species and a reactive surface at $x=0$ is considered. Species 1 and 2 are present in the fluid and on the surface and have diffusivities of unity. The concentrations of these species are fixed at $x=1: c^{(1)}(1)=1$ and $c^{(2)}(1)=0$. Species 3 is present on the surface but not in the fluid and has effectively zero diffusivity. The following conditions apply on the reactive surface at $x=0$ :

$$
-\frac{d c^{(1)}}{d x}=\gamma\left(c^{(3)}-c^{(1)}\right),-\frac{d c^{(2)}}{d x}=\gamma\left(c^{(3)}-c^{(2)}\right), 0 \approx \gamma\left(c^{(1)}-c^{(3)}\right)+\gamma\left(c^{(2)}-c^{(3)}\right)
$$

where $\gamma>0$ is the stiffness parameter, effectively the ratio of the reaction rate to the diffusion rate. These equations correspond to two surface reactions: “ 1 " $\leftrightarrow$ " 3 " and " 3 " $\leftrightarrow$ " 2 ", both with forward and backward rate parameters of $\gamma$. This system has the following analytical solution:

$$
c^{(1)}(x)=1-\Gamma(1-x), c^{(2)}(x)=\Gamma(1-x), c^{(3)}(0)=\frac{1}{2}, \text { where } \Gamma=\frac{\gamma}{2(1+\gamma)} .
$$

Figure 2.1 shows FIDAP results using the method outlined above for $\gamma=99$. A species 3 diffusivity of $10^{-7}$ is used which appears to be sufficiently small. Note that in both cases the analytical results for $c^{(1)}(x), c^{(2)}(x)$, and $c^{(3)}(0)$ are obtained despite setting $c^{(3)}$ equal to 1 (rather than the expected surface value of $1 / 2$ ) away from the reactive surface. Additional simulations employing different $\gamma$ values (both larger and smaller) are also found to reproduce the analytical results identically. 

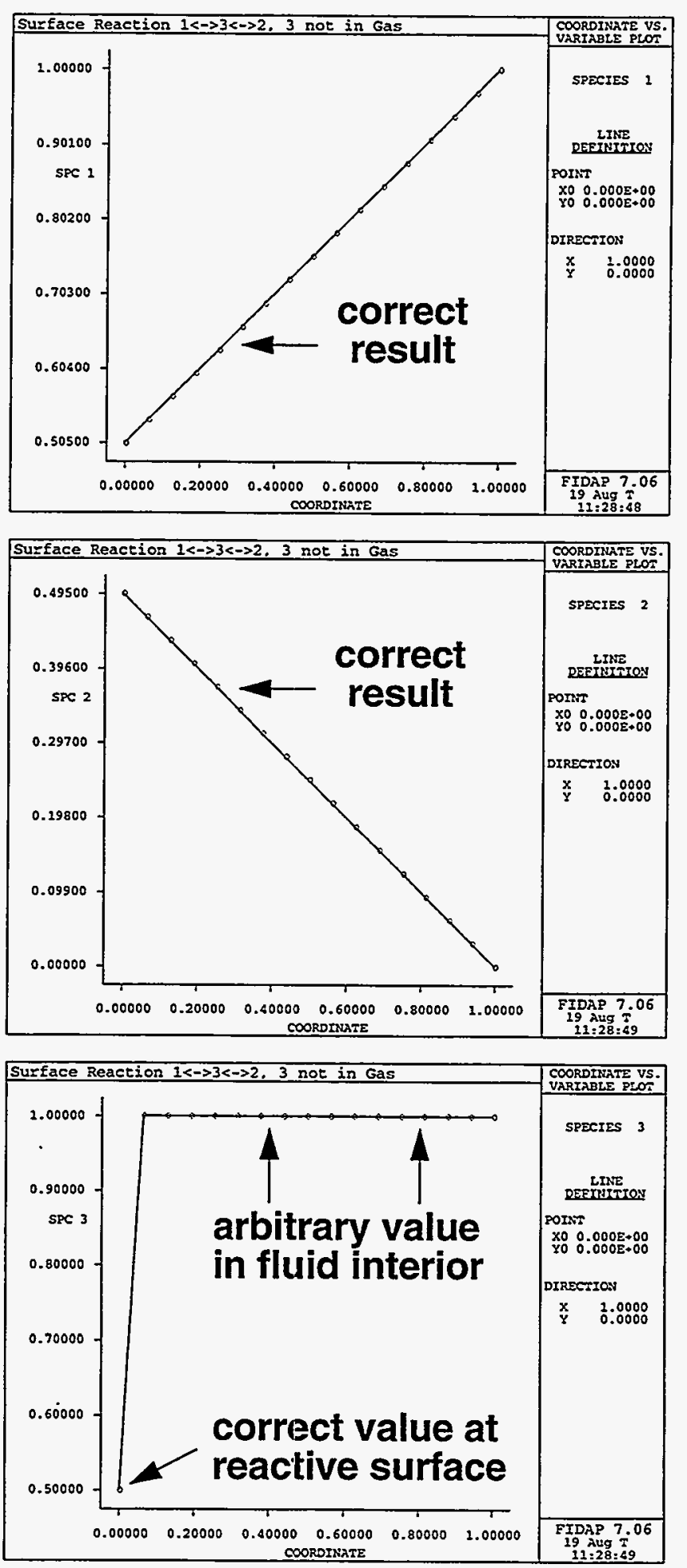

Figure 2.1. One-dimensional reaction-diffusion problem for $\gamma=99$. 


\subsection{Two-Dimensional Example}

To illustrate how this method performs for multidimensional problems, simulations are performed using the two-dimensional flow over a backward-facing step, with a reactive surface located upstream of the step, on which the chemistry used in the previous example is applied but with $\gamma=1$. An advection-diffusion analysis is performed for this case: the flow field is selved for first, and the chemistry is solved for subsequently. Table 2.1 provides geometric information, material properties, and boundary conditions for the simulations. The following analytical results hold for this case:

$$
c^{(1)}(x, y)+c^{(2)}(x, y)=1 \text { and } c^{(3)} \text { (reactive surface) }=1 / 2 .
$$

Figure 2.2 shows the computational mesh and contour plots of the streamlines, temperature, and species concentrations. The species 1 and species 2 contour locations are identical and their values sum to unity, indicating satisfaction of the analytical result. The species 3 contours depart from unity only at the reactive surface, on which $c^{(3)}$ is identical to the analytical value of $1 / 2$.

Table 2.1. Information for the surface-species backward-facing step simulations.

\begin{tabular}{|l|l|}
\hline \multicolumn{1}{|c|}{ Quantity } & \multicolumn{1}{c|}{ Value } \\
\hline \hline Channel length upstream & 4, from $x=-4$ to $x=0$ \\
\hline Channel length downstream & 6, from $x=0$ to $x=6$ \\
\hline Step height & 0.5, from $y=-0.5$ to $y=0$ \\
\hline Channel height upstream & 0.5, from $y=0$ to $y=0.5$ \\
\hline Channel height downstream & 1, from $y=-0.5$ to $y=0.5$ \\
\hline Heated region & $-2 \leq x \leq 0, y=0$ \\
\hline Density & 1 \\
\hline Viscosity & 0.01 \\
\hline Specific heat & 1 \\
\hline Thermal conductivity & $1 / 71$ \\
\hline Species $1,2,3$ capacities & $1,1,0$ (convection term removed) \\
\hline Species $1,2,3$ diffusivities & $1 / 71,1 / 71,10^{-7} / 71$ \\
\hline Velocity at inlet, reactive surface, other walls & $\left(12 y-24 y^{2}, 0,0\right),(0,0,0),(0,0,0)$ \\
\hline Temperature at inlet, reactive surface, other walls & $1,2,1$ \\
\hline Species 1,2 concentrations at inlet & 1,0 \\
\hline Species fluxes at reactive surface, other walls & chemistry, 0 \\
\hline Species 3 concentration away from reactive surface & 1 (arbitrary) \\
\hline Velocity, temperature, species at outlet & natural boundary conditions \\
\hline & \\
\hline
\end{tabular}



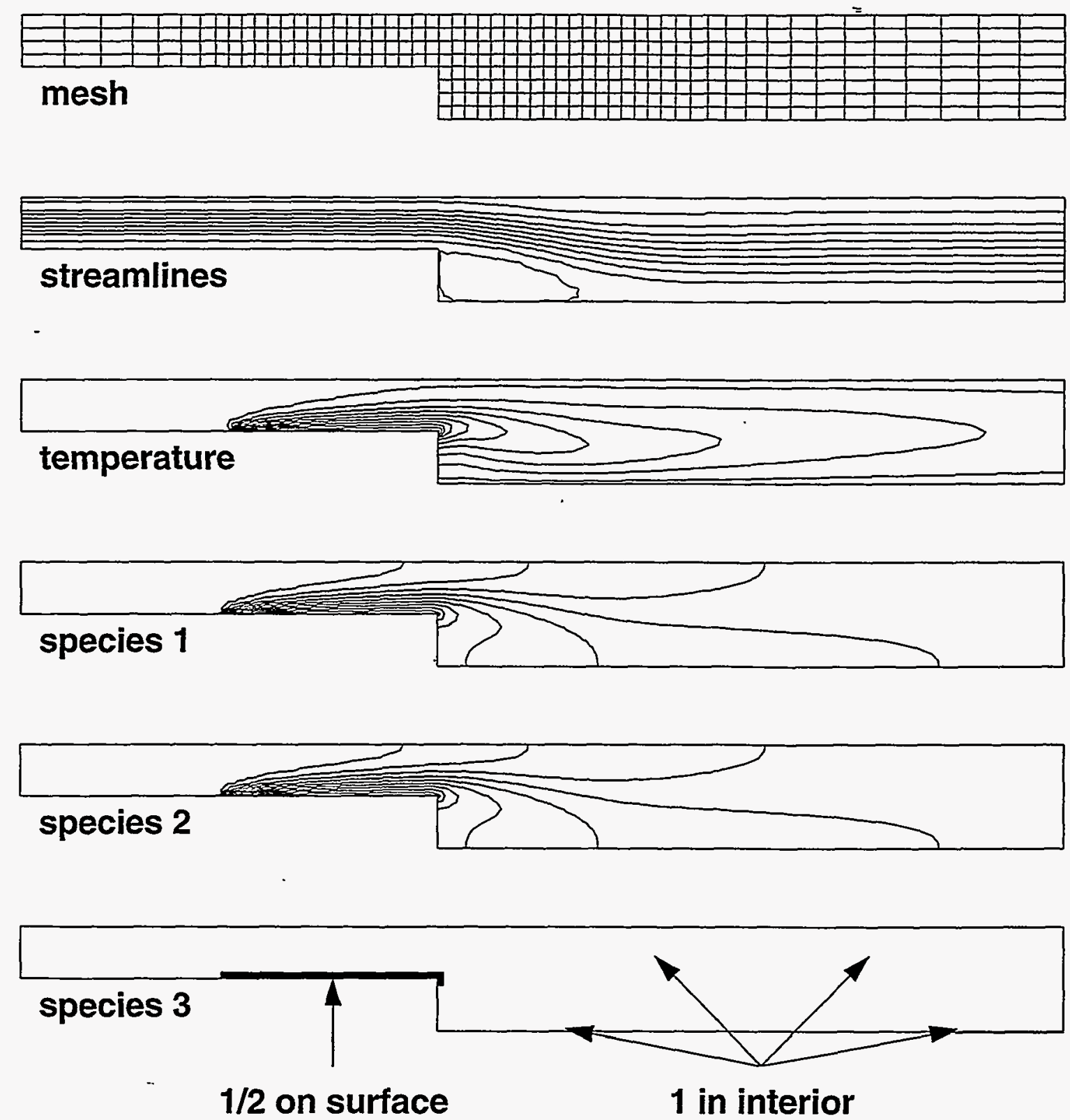

Figure 2.2. Backward-facing step simulation with surface species and reactions. 


\section{Full-Jacobian Approach for Stiff Chemistry}

\subsection{Method}

In many semiconductor-processing applications involving gas flow and chemistry, "stiff" chemistry is encountered: the chemistry is orders of magnitude faster than diffusive or convective transport, so the stiffness parameter $\gamma$, which describes the ratio of the chemical reaction rate to the transport rate (a Damköhler number), becomes large. When this occurs, how the chemical-reaction source terms are treated affects the convergence behavior of a solution algorithm. In what follows, the implementation in FIDAP of a full-Jacobian treatment of the chemical-reaction source terms is described, and examples are presented demonstrating its effect on convergence behavior.

The physical system considered consists of chemical species $c^{(m)}$ (some may be surface species) which are transported and can react in the fluid and on reactive surfaces according to

$$
\begin{gathered}
\rho C^{(m)} \vec{u} \cdot \nabla c^{(m)}=\nabla \cdot\left(\rho \alpha^{(m)} \nabla c^{(m)}\right)+S^{(m)}\left(\left\{c^{(n)}\right\}\right) \text { in the fluid, } \\
-\rho \alpha^{(m)} \hat{n} \cdot \nabla c^{(m)}+c^{(m)} s_{t}=s^{(m)}\left(\left\{c^{(n)}\right\}\right), \rho \vec{u} \cdot \hat{n}=s_{t}, s_{t}=\sum_{m} s^{(m)} \text { on the surface, }
\end{gathered}
$$

where the fluid-phase and surface chemical-reaction source terms $S^{(m)}$ and $s^{(m)}$ for $c^{(m)}$ can depend on all the other species concentrations $\left\{c^{(n)}\right\}$. The corresponding discretized version of this system can be expressed as

$$
K^{(m)} \delta c^{(m)}-\delta S^{(m)}=-\left[K^{(m)} c^{(m)}-S^{(m)}\right],
$$

where $K^{(m)}$ is the diffusive-convective stiffness matrix evaluated using quantities from the previous iteration, $S^{(m)}$ is the chemical-reaction source-term vector evaluated using quantities from the previous iteration, $\delta c^{(m)}$ is the vector of species concentration updates, and $\delta S^{(m)}$ is the vector of chemical-reaction source-term updates.

To enable solution of this system, a linear form must be provided for $\delta S^{(m)}$ in terms of $\left\{\delta c^{(n)}\right\}$. Several treatments are possible, including the following:

$$
\begin{gathered}
\delta S^{(m)} \rightarrow 0 \text { (successive substitution), } \\
\delta S^{(m)} \rightarrow\left(\partial S^{(m)} / \partial c^{(m)}\right) \delta c^{(m)} \text { (diagonal Jacobian), } \\
\delta S^{(m)} \rightarrow \sum_{n}\left(\partial S^{(m)} / \partial c^{(n)}\right) \delta c^{(n)} \text { (full Jacobian), }
\end{gathered}
$$

where $\partial S^{(m)} / \partial c^{(n)}$ is the Jacobian derivative matrix evaluated at the previous iteration. Of these three treatments, only the full-Jacobian approach is appropriate for problems with stiff chemistry. A disadvantage of the full-Jacobian approach is that the resultant matrix equations for the different species are strongly cross-coupled by the off-diagonal terms, which renders solutions difficult to obtain with segregated algorithms. However, although decoupled and thus amenable to segregation, the other two choices often are unstable or, if stable, converge at extremely slow rates. In the next chapter, a method is presented for combining the full-Jacobian treatment of stiff chemistry with a segregated approach. 
FIDAP has been modified to allow solution of the coupled linear system using the full-Jacobian approach to represent the chemical-reaction source terms:

$$
\begin{gathered}
K^{(m)} \delta c^{(m)}-\sum_{n}\left(\partial S^{(m)} / \partial c^{(n)}\right) \delta c^{(n)}=-\left[K^{(m)} c^{(m)}-S^{(m)}\right] \\
c^{(m)} \rightarrow c^{(m)}+\delta c^{(m)} .
\end{gathered}
$$

This implementation has been performed in FIDAP for both fluid-phase and surface chemical reactions. Both the Arrhenius-parameter (CONSTANT keyword on the REACTION card in FIPREP) and user-subroutine (SUBROUTINE keyword on the REACTION card in FIPREP to access the USRRXN subroutine) methods [1] can be used to specify reaction parameters. The user also has the option of either numerically differencing the source terms to determine the Jacobian matrix or entering the Jacobian matrix directly via a user subroutine.

This approach has been tested on a wide variety of cases, including some with up to four chemical species, various combinations of fluid-phase, fluid-surface, and surface chemical species, linear and nonlinear chemical-reaction source terms, isothermal and coupled-thermal problems, and advectiondiffusion and fully coupled flow-thermal-chemical problems. In all cases examined, known analytical results are always reproduced, and good convergence behavior typical of Newton-Raphson methods is observed without the use of nonzero acceleration factors and independent of whether numerical or analytical Jacobian matrices are used, even for stiff problems.

\subsection{Two-Dimensional Example}

To illustrate the full-Jacobian approach, chemically reacting flow over a two-dimensional backwardfacing step is considered. As before, a heated reactive surface upstream of the step is included. Figure 3.1 shows the convergence history for the simulation discussed below, Figure 3.2 shows a schematic diagram of the geometry and the mesh used in the simulations, and Table 3.1 shows geometric information, material properties, and boundary conditions. The velocity and temperature fields are weakly coupled by virtue of the temperature-dependent material properties, so solving this problem demonstrates that the full-Jacobian approach is not limited only to advection-diffusion problems. Four species are considered: species 1, 2, and 4 in the fluid, and species 3 on the reactive surface. Heat is released as all reactions proceed, and all reaction rates and the heat production rate are temperature-dependent. The fluid-phase and surface reaction rates, $S^{(m)}$ and $s^{(m)}$, and the fluidphase and surface heat production rates, $Q$ and $q$, are given by:

$$
\begin{gathered}
S^{(1)}=-S^{(2)}=\gamma \exp \left(-\frac{E_{1}}{T}\right)\left(c^{(2)}-c^{(1)}\right), S^{(4)}=0, \\
Q=-H S^{(1)}, \\
s^{(1)}=s^{(2)}=\gamma T^{\beta} \exp \left(-\frac{E_{2}}{T}\right)\left(c^{(3) 2}-c^{(1)} c^{(2)}\right), s^{(4)}=\gamma T^{\beta} \exp \left(-\frac{E_{2}}{T}\right)\left(c^{(3)}-c^{(4)}\right), \\
s^{(3)}=-\left(s^{(1)}+s^{(2)}+s^{(4)}\right), \\
q=-H s^{(1)},
\end{gathered}
$$

where the Arrhenius parameters are $\gamma=10^{3}$ (very stiff), $E_{1}=1, E_{2}=10, \beta=0.75$, and $H=10$. 
Table 3.1. Information for the full-Jacobian backward-facing step simulations.

\begin{tabular}{|l|l|}
\hline \multicolumn{1}{|c|}{ Quantity } & \multicolumn{1}{c|}{ Value } \\
\hline \hline Geometry - & identical to Table 2.1 = \\
\hline Density & 1 \\
\hline Viscosity & $\mu(T)=T^{0.7} / 100$ \\
\hline Specific heat & 1 \\
\hline Thermal conductivity & $k(T)=T^{0.7} / 71$ \\
\hline Species $1,2,3,4$ capacities & $1,1,0,1($ convection term removed) \\
\hline Species $1,2,3,4$ diffusivities & $\alpha^{(1,2,4)}(T)=T^{0.7} / 71, \alpha^{(3)}(T)=10^{-7} T^{0.7} / 71$ \\
\hline Velocity at inlet, reactive surface, other walls & $\left(12 y-24 y^{2}, 0,0\right),(0,0,0),(0,0,0)$ \\
\hline Temperature at inlet, reactive surface, other walls & $1,5,1$ \\
\hline Species $1,2,4$ concentrations at inlet & $0.5,0.5,0$ \\
\hline Species fluxes at reactive surface, other walls & chemistry, 0 \\
\hline Velocity, temperature, species at outlet & natural boundary conditions \\
\hline
\end{tabular}

Several analytical results are available for this case. Since $s^{(1)}=s^{(2)}$, the surface reactions result in equal changes of species 1 and species 2. Thus, since $c^{(1)}=c^{(2)}$ at the inlet, it is found that $c^{(1)}=c^{(2)}$ and $S^{(1)}=S^{(2)}=0$ everywhere in the fluid. Species 4 is created at the heated reactive surface and is transported out into the fluid phase. Since $S^{(1)}+S^{(2)}+S^{(4)}=0$ in the fluid phase and $s^{(1)}+s^{(2)}+s^{(4)}=0$ at the surface by virtue of $s^{(1)}+s^{(2)}+s^{(3)}+s^{(4)}=0$ and $s^{(3)}=0$ (species 3 is a surface species), it can be shown that in the fluid $c^{(1)}+c^{(2)}+c^{(4)}=1$, as expected. Although not obtained due to the finite rate of the chemical reactions $(\gamma<\infty)$, if equilibrium were obtained at the reactive surface, then $c^{(4)}=c^{(3)}=\left(c^{(1)} c^{(2)}\right)^{1 / 2}$ would be observed, which with the above constraints implies values of $1 / 3$ for each of $c^{(1)}, c^{(2)}, c^{(3)}$, and $c^{(4)}$ along the reactive surface as $\gamma \rightarrow \infty$. The fact that the sum of these four "mass fractions" exceeds unity does not pose a difficulty: $c^{(3)}$ should not be included in the sum of mass fractions for the fluid phase since species 3 exists only on the reactive surface. Thus, as discussed previously, the precise meaning of the $c^{(3)}$ value depends on the interpretation assigned to $c^{(3)}$ when developing the particular expressions for the surface chemicalreaction source terms $s^{(m)}$.

Since for highly nonlinear problems the starting guess for the fields can play a very important part in finding the solution, a reasonable initial guess for the velocity field is generated by solving the corresponding flow and thermal problem without chemistry in the same geometry with the same velocity and temperature boundary conditions (no temperature dependence on the viscosity is used). The velocity field from this simulation serves as the initial guess for the velocity field in the coupled flow-thermal-chemistry problem although the initial temperature field for the latter is set to a constant value of 3 at all interior nodes. The initial concentrations of species 1 and species 2 are set to the fluid-phase equilibrium concentration of 0.5 , and the initial concentrations of species 3 and species 4 are both set to 0.1 on the interior nodes. 
Two simulations are performed: one in which the reactions are specified using the CONSTANT keyword on the REACTION card with the Jacobian terms calculated analytically, and the other in which the reactions are specified using the SUBROUTINE keyword on the REACTION card with the Jacobian terms calculated by numerical differencing. In both cases, acceleration factors of zero are employed, and convergence of all fields occurs in 9 iterations (no appreciable differences are found between the results). Figure 3.1 shows the norms of the relative errors for all degrees of freedom as a function of iteration number. This plot shows a smooth transition from a low convergence rate to a significantly higher convergence rate with increasing iteration number, which is characteristic of Newton-Raphson approaches. Applying the original algorithm (without the full-Jacobian approach) to this problem does not yield a solution for $\gamma$ values much larger than unity. The flow, thermal, and concentration results of the first simulation are shown in Figure 3.2. In agreement with the analytical results, it is observed that $c^{(1)}=c^{(2)}$ and $c^{(1)}+c^{(2)}+c^{(4)}=1$ in the fluid. On the reactive surface, due to the large value of $\gamma$, only slight departures are seen from the equilibrium values of $c^{(1)}=c^{(2)}=c^{(3)}=c^{(4)}=1 / 3$.

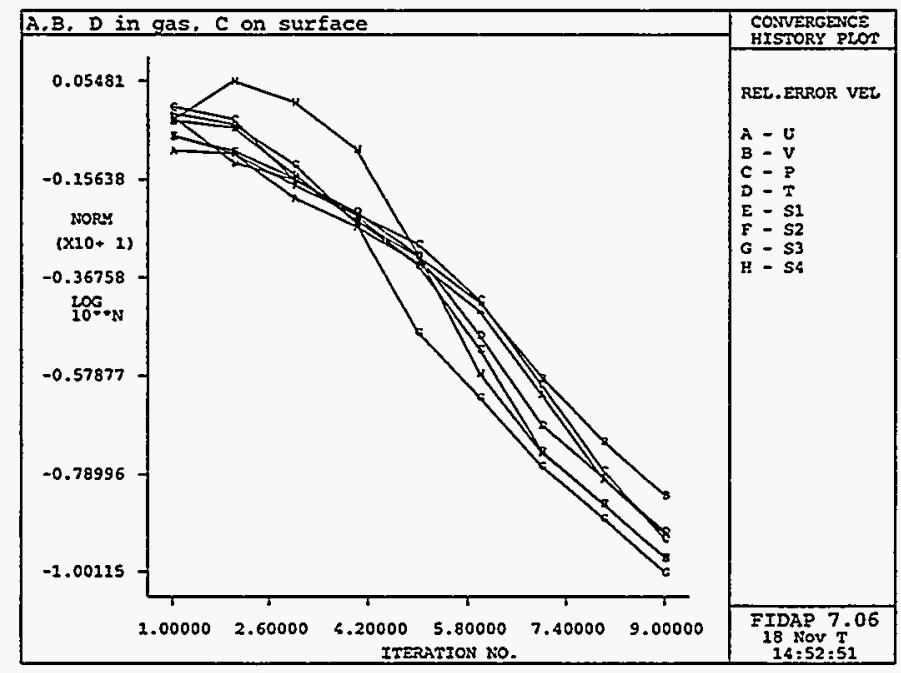

Figure 3.1. Convergence history for the full-Jacobian backward-facing step simulation. 

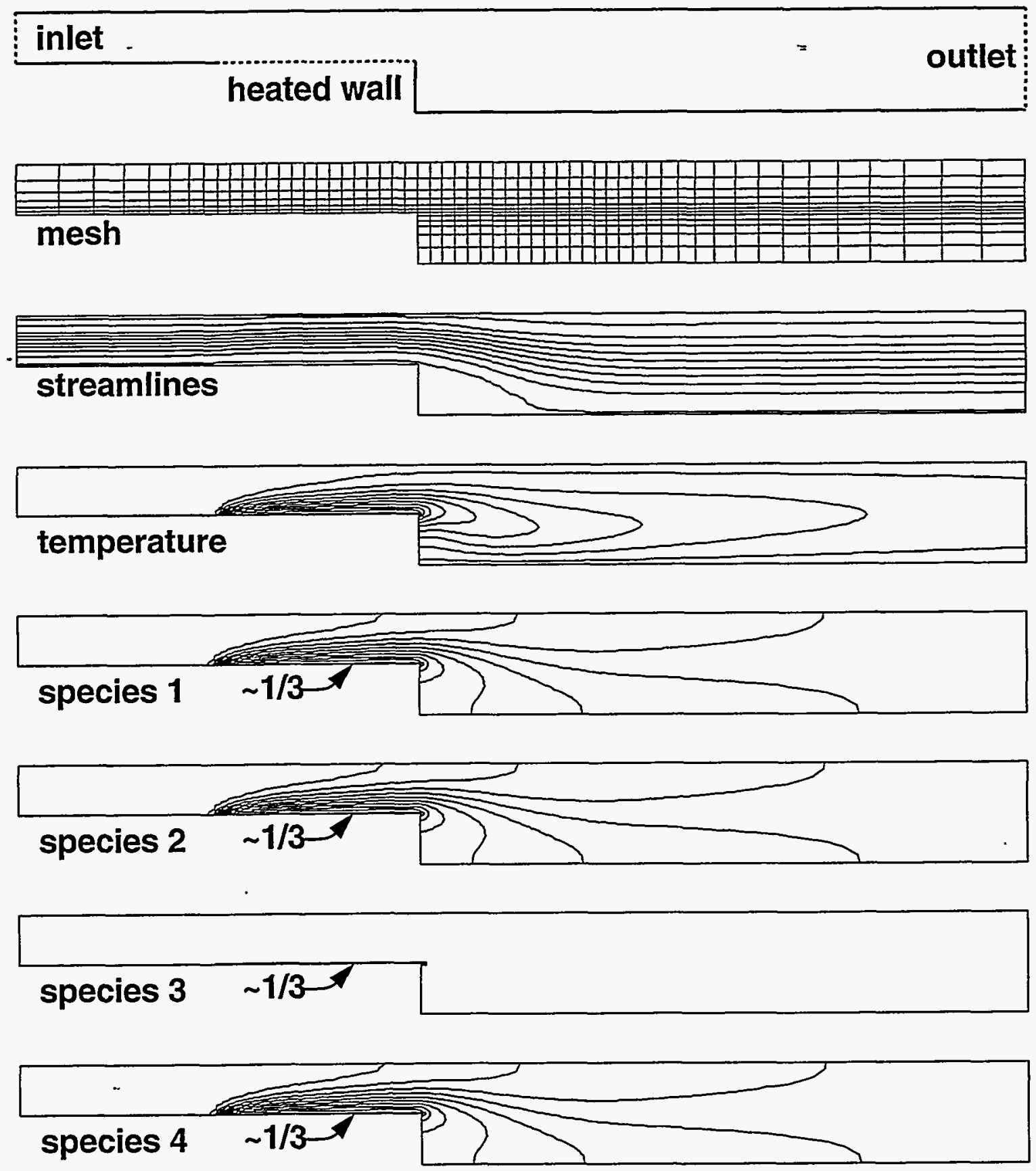

Figure 3.2. Backward-facing step simulation with full-Jacobian approach for $\gamma=10^{3}$. 


\section{Segregated Solution Approach for Stiff Chemistry}

\subsection{Method}

In the previous chapter, it was shown that incorporating a full Jacobian of the chemical-reaction source terms into the matrix equation improves the convergence behavior of the solution algorithm. However, the cost associated with this approach is that the species-transport equations are strongly cross-coupled: the effect of the variations of all species must be simultaneously included in each species transport equation. Incorporation of this cross-species coupling appears to preclude the use of segregated algorithms to obtain solutions since segregated approaches effectively consider all species but one to be constant during each portion of the iteration. However, a segregated algorithm is often necessary to maintain a manageable problem size, particularly for the large three-dimensional multispecies problems that can be encountered in semiconductor-processing applications. Thus, an algorithm is desired that is both chemically coupled and segregated, which appear to be contradictory goals. Nevertheless, in the remainder of this section, one approach is outlined that maintains the strong cross-species chemical coupling in an approximate fashion yet is segregated in implementation. This stiff segregation algorithm has been implemented in FIDAP, and examples are presented to illustrate its behavior.

To outline this method, consider a chemistry problem involving three species, labelled "1", "2", and " 3 ". Suppose for convenience that the boundary conditions are such that at each node either all or none of the species concentrations are known (this can be relaxed straightforwardly, but the notation becomes more cumbersome). Then the global linear system that must be solved can be written schematically as

$$
\left[\begin{array}{lll}
M^{(11)} & M^{(12)} & M^{(13)} \\
M^{(21)} & M^{(22)} & M^{(23)} \\
M^{(31)} & M^{(32)} & M^{(33)}
\end{array}\right]\left[\begin{array}{l}
\delta c^{(1)} \\
\delta c^{(2)} \\
\delta c^{(3)}
\end{array}\right]=\left[\begin{array}{l}
V^{(1)} \\
V^{(2)} \\
V^{(3)}
\end{array}\right],
$$

where $N_{n}$ is the number of nodes at which the $N_{s_{2}}$ species concentrations are unknown and the entries $M^{(m n)}$ are $N_{n} \times N_{n}$ banded matrices, of $\mathrm{O}\left(N_{n}^{s_{2 / 3}}\right)$ bandwidth for three-dimensional problems. The vectors $\delta c^{(m)}$ and $V^{(m)}$ represent the updates to species concentration unknowns and the righthand side vectors for species $m$. In segregation as it is generally applied, all of the off-diagonal matrices are zeroed to yield

$$
\left[\begin{array}{ccc}
M^{(11)} & 0 & 0 \\
0 & M^{(22)} & 0 \\
0 & 0 & M^{(33)}
\end{array}\right]\left[\begin{array}{l}
\delta c^{(1)} \\
\delta c^{(2)} \\
\delta c^{(3)}
\end{array}\right]=\left[\begin{array}{l}
V^{(1)} \\
V^{(2)} \\
V^{(3)}
\end{array}\right],
$$

which as previously discussed is inappropriate when stiff chemical reactions are present. In the stiff segregated method, it is necessary to select an "active" degree of freedom, the species that will be updated during this portion of the iteration (all other species are "inactive"). For convenience, species 1 is selected. All submatrices except those that multiply the active degree of freedom are summarily truncated to only their diagonal elements, leaving the approximate system 


$$
\left[\begin{array}{lll}
M^{(11)} & D^{(12)} & D^{(13)} \\
M^{(21)} & D^{(22)} & D^{(23)} \\
M^{(31)} & D^{(32)} & D^{(33)}
\end{array}\right]\left[\begin{array}{l}
\delta c^{(1)} \\
\delta c^{(2)} \\
\delta c^{(3)}
\end{array}\right]=\left[\begin{array}{c}
V^{(1)} \\
V^{(2)} \\
V^{(3)}
\end{array}\right]
$$

where $D^{(m n)}$ is the diagonal portion of $M^{(m n)}$. Now consider the equation associated with the species 1 degree of freedom at node $i$

$$
\sum_{j} M_{i j}{ }^{(11)} \delta c_{j}{ }^{(1)}+D_{i i}{ }^{(12)} \delta c_{i}^{(2)}+D_{i i}{ }^{(13)} \delta c_{i}^{(3)}=V_{i}^{(1)}
$$

Here, $M_{i j}{ }^{(11)}$ is entry $j$ of row $i$ of the corresponding submatrix. In order to have a segregated scheme, it is necessary to eliminate the inactive species unknowns $\delta c_{i}^{(2)}$ and $\delta c_{i}^{(3)}$ from this equation. To accomplish this, the other two equations associated with node $i$ are used:

$$
\begin{aligned}
& \sum_{j} M_{i j}{ }^{(21)} \delta c_{j}^{(1)}+D_{i i}{ }^{(22)} \delta c_{i}^{(2)}+D_{i i}{ }^{(23)} \delta c_{i}^{(3)}=V_{i}^{(2)} \\
& \sum_{j} M_{i j}^{(31)} \delta c_{j}^{(1)}+D_{i i}^{(32)} \delta c_{i}^{(2)}+D_{i i}^{(33)} \delta c_{i}^{(3)}=V_{i}^{(3)}
\end{aligned}
$$

These latter two equations are used to find $\delta c_{i}{ }^{(2)}$ and $\delta c_{i}{ }^{(3)}$ as functions of the species 1 vector $\delta c^{(1)}$ and to eliminate them from the first equation. Note that operations on a $2 \times 2$ matrix rather than an $N_{n} \times N_{n}$ matrix are required: $i$ enters parametrically. The identical process is repeated for all other nodes, and the end result is a segregated matrix equation for species 1 :

$$
\tilde{M}^{(11)} \delta c^{(1)}=\tilde{V}^{(1)},
$$

where $\tilde{M}^{(11)}$ is a modified species 1 stiffness matrix containing contributions from $M^{(21)}, M^{(31)}$, and all of the diagonalized matrices. Similarly, $\tilde{V}^{(1)}$ is a modified right-hand side vector. The contributions from these off-diagonal terms stabilize the solution scheme for stiff chemistry when the magnitude of the off-diagonal terms becomes comparable to or larger than the on-diagonal terms.

A few points about implementation of the above approach require some elaboration. In particular, the previous discussion appears to assume that the entire global stiffness matrix is available for use. Of course, this is not the case when solving a problem for which a segregated algorithm is necessary (that is the point of using a segregated algorithm). Consider how to implement the stiff segregation solution algorithm from the standpoint of each element's local stiffness matrix, that is, during rather than following global assembly. The most obvious approach is to attempt to apply the algorithm to each local stiffness matrix prior to its assembly into the global matrix. Unfortunately, because the algorithm requires both a matrix inversion and multiplication of matrix elements, this approach will not work because the summations of the contributions to each node from its surrounding elements will not distribute properly, and the result will be incorrect. The $D_{i i}^{(m n)}$ and related quantities (but not the $M_{i j}^{(m n)}$ ) must be computed on a global basis, that is, taking into account the contributions from all elements, before stiff segregation can be applied during assembly. Therefore, a three-pass process is employed. During the first pass, all local stiffness matrices are computed and the appropriate quantities are extracted from them and accumulated. In a second pass, these accumulated quantities are modified to yield quantities employed in the subsequent assembly of the modified global matrix, which occurs during the third pass. The steps that occur in this three-pass process are delineated below and have been implemented in FIDAP. 


\subsection{Implementation Steps}

For $N$ species, with species $s$ active and the remaining species inactive, apply the following steps:

Step 1: Loop over all elements.

A) Compute the local stiffness matrix for each element $e$.

B) Extract $D_{i i}{ }^{(m n)(e)}$ from each matrix and accumulate it into $D_{i i}{ }^{(m n)}$. The local stiffness matrices are not assembled into the global matrix during this pass.

Step 2: Loop over all nodes.

A) For each node $i$, invert $D_{i i}{ }^{(m n)}$ over $(m n)$ to obtain $Z_{i i}{ }^{(n m)}$ according to:

$$
Z_{i i}^{(n m)}=\left(D_{i i}{ }^{(m n)}\right)^{-1}, m \neq s, n \neq s .
$$

B) Determine $R_{i}^{(s m)}$ from these two matrices according to:

$$
R_{i}^{(s m)}=-\sum_{n \neq s} D_{i i}^{(s n)} Z_{i i}^{(n m)}, m \neq s
$$

Step 3: Loop over all elements.

A) Compute the local stiffness matrix and right hand-side vector for each element $e$ and modify them according to:

$$
\begin{gathered}
\tilde{M}_{i j}^{(s s)(e)}=M_{i j}^{(s s)(e)}+\sum_{m \neq s} R_{i}^{(s m)} M_{i j}^{(m s)(e)}, \\
\tilde{V}_{i}^{(s)(e)}=V_{i}^{(s)(e)}+\sum_{m \neq s} R_{i}^{(s m)} V_{i}^{(m)(e)} .
\end{gathered}
$$

B) Assemble the modified local stiffness matrices and right hand-side vectors (given above) into their global counterparts.

Step 4: Solve the global matrix system $\tilde{M}^{(s s)} \delta c^{(s)}=\tilde{V}^{(s)}$.

Step 5: Update the species $s$ concentrations $c^{(s)} \rightarrow c^{(s)}+\delta c^{(s)}$.

Step 6: Increment the active species and return to Pass 1.

A single iteration is completed when all species have been the active species once.

\subsection{Additional Considerations}

As discussed above, the stiff segregation algorithm differs from the original segregated algorithm in FIDAP by requiring additional computational steps for additional quantities. Therefore, different amounts of memory are needed for the same physical problem, and individual iterations are longer. Also, as for any segregated strategy, stability and approach to convergence become important issues.

The additional memory requirements to implement this algorithm are acceptable. During the first pass, $N_{n}\left(N_{s}-1\right)^{2}$ memory locations need to be allocated to store the $D_{i i}{ }^{(m n)}$ matrices, where $N_{s}$ is 
the number of species and $N_{n}$ is the number of nodes. For typical three-dimensional problems, this is comparable to or smaller than the $N_{n}^{5 / 3}$ memory locations needed to store the global matrix if it has a bandwidth of $N_{n}^{2 / 3}$. After the second pass, only $N_{n}\left(N_{s}-1\right)$ memory locations are needed to store the back substitution coefficients $R_{i}^{\left({ }^{(s m)}\right.}$. For both passes combined, this amount of memory is equivalent to $\left(N_{s}-1\right)$ additional solution vectors.

A second issue is the additional computational time associated with the algorithm. The fact that there are two sweeps through the element list does not necessarily mean that the computational time is doubled. In general, the time for assembly of the global matrix is a small fraction of the time required to solve the corresponding linear system. Hence, making essentially two assembly passes should not dramatically affected the running time. The other issue is the amount of time needed to invert $N_{n}\left(N_{s}-1\right) \times\left(N_{s}-1\right)$ matrices during the second pass. Roughly speaking, the number of operations needed to invert a full $\left(N_{s}-1\right) \times\left(N_{s}-1\right)$ matrix scales as $\left(N_{s}-1\right)^{3}$. Therefore, the operations needed for the second pass is $N_{n}\left(N_{s}-1\right)^{3}$, whereas solving a global matrix of bandwidth $N_{n}^{2 / 3}$ requires roughly $N_{n}^{7 / 3}$ operations. Consequently, the relative time between pass 2 and pass 3 should scale as $\left(N_{s}-1\right)^{3} / N_{n}^{4 / 3}$. A three-dimensional problem typically contains at least $N_{n}=20^{3}=8000$ nodes, and the maximum number of species allowed in FIDAP is $N_{s}=15$. In this case, the time for the second pass would be only a small fraction of the time for the third pass. This fraction would increase for problems with fewer nodes, but for these problems it would probably be better to use the direct solver.

Convergence behavior and stability for the stiff segregated algorithm are typical of segregated algorithms. First, convergence is still rather slow although much better than the original segregated algorithm, as shown below. Second, the method becomes unstable when convection dominates either diffusion or chemistry. This is because the convection portion of the stiffness matrix generally has zeroes for diagonal entries. In the former case, this difficulty can be circumvented by selecting an upwinding factor of unity, which places entries of the appropriate size on the diagonal. In the latter case, the original segregated solver should be used.

\subsection{One-Dimensional Example}

To illustrate the above approach, a one-dimensional diffusion problem (no convection) with a reactive surface at $x=1$ is considered. Two species, denoted " 1 " and " 2 ", are present in the fluid and on the surface and have diffusivities of unity. The concentrations of these species are fixed at $x=0$ : $c^{(1)}(0)=1$, and $c^{(2)}(0)=0$. The following conditions apply on the reactive surface at $x=1$ :

$$
\frac{d c^{(1)}}{d x}=\gamma\left(c^{(2)}-c^{(1)}\right), \frac{d c^{(2)}}{d x}=\gamma\left(c^{(1)}-c^{(2)}\right),
$$

where $\gamma>0$ is the stiffness parameter. This system has the following analytical solution:

$$
c^{(1)}(x)=1-\Gamma x, c^{(2)}(x)=\Gamma x, \text { where } \Gamma=\frac{\gamma}{1+2 \gamma} .
$$

This problem is a good test case because it can be solved using both the direct and segregated approaches and has an analytical solution. Due to the linearity of the source term, the full-Jacobian direct approach yields a converged solution to the analytical result for arbitrarily large $\gamma$ values in exactly 2 iterations, independent of the initial guess (really only 1 iteration is required, but an additional iteration is required to "verify" that the solution has converged). Application of the stiff segregated approach yields the analytical result, but with slower convergence. Convergence of the 
original segregated method depends on how the reactions are specified. If the Arrhenius-parameter method is employed, the original method uses the diagonal-Jacobian approach and appears to converge, but at an extremely slow rate. If the user-subroutine method is employed, the original method uses the successive-substitution approach and diverges for all values. of $\gamma$ much greater than unity. As an example, Figure 4.1 shows the $\gamma=10^{3}$ solution achieved after 100 iterations of the stiff segregated solver, which agrees with the analytical result to 5 decimal places, and the solution achieved after 1000 iterations of the original segregated solver (using the diagonal-Jacobian approach), which differs substantially from the analytical result.
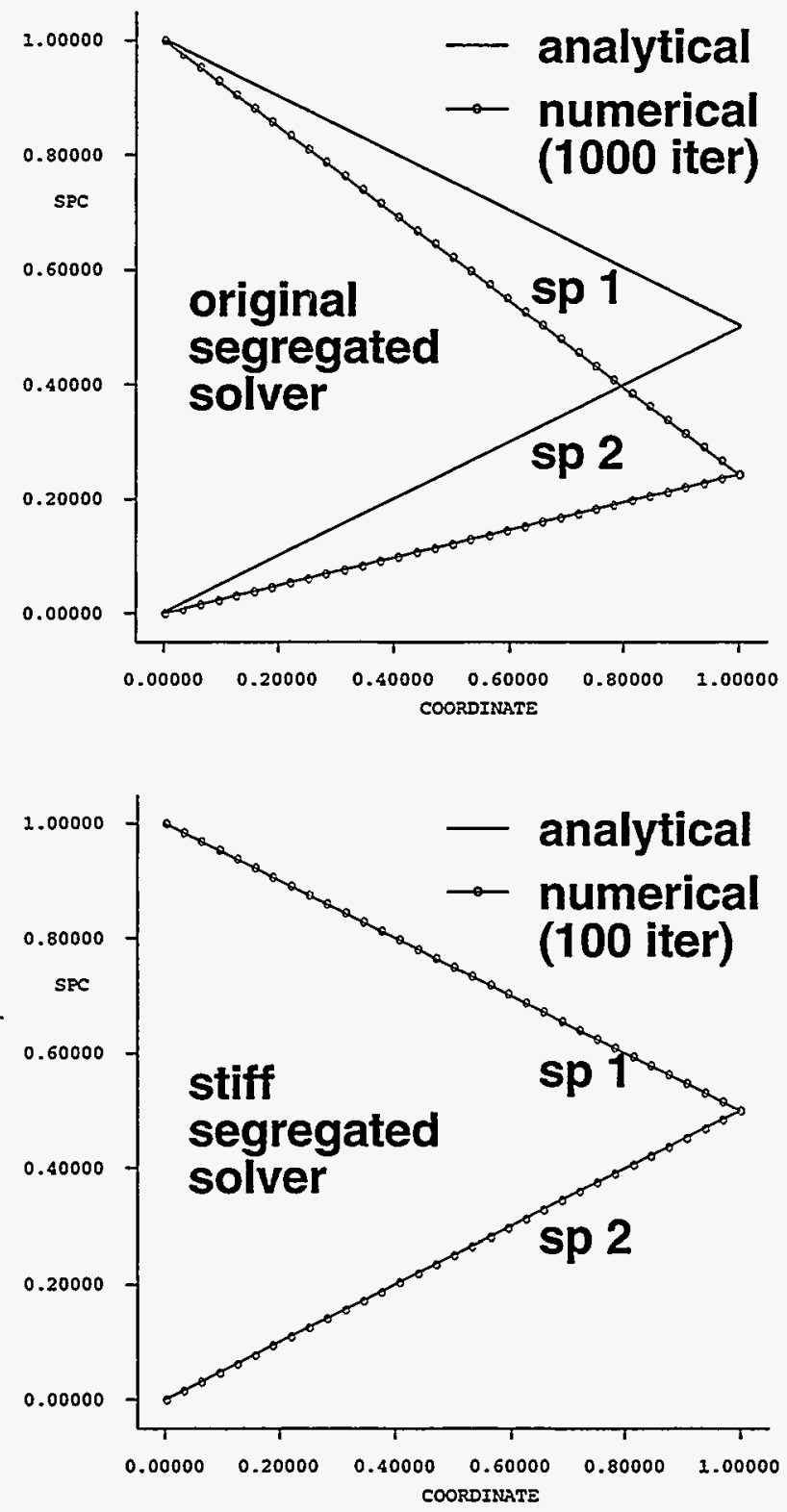

Figure 4.1. One-dimensional reaction-diffusion problem with $\gamma=10^{3}$. 


\subsection{Three-Dimensional Example}

To illustrate the applicability of the stiff segregated solver to three-dimensional chemically reacting flows, three-dimensional flow of a reactive three-species mixture (species 1,2, and 4) over a backwardfacing step is considered (the chemistry is taken to be dilute and does not back-couple to the flow field). Just upstream of the step is a heated reactive surface, on which species 1,2 , and 4 , can react directly with each other and also via another species (species 3 ), which exists only on the reactive surface. The fluid-phase reaction rates $S^{(m)}$ and the surface reaction rates $s^{(m)}$ are given below:

$$
\begin{gathered}
S^{(1)}=-S^{(2)}=\gamma \exp \left(-\frac{E_{1}}{T}\right)\left(c^{(2)}-c^{(1)}\right), S^{(4)}=0, \\
s^{(1)}=\frac{1}{2} s^{(2)}=\gamma \exp \left(-\frac{E_{2}}{T}\right)\left(c^{(3) 2}-c^{(1)} c^{(2)}\right), s^{(4)}=\gamma \exp \left(-\frac{E_{2}}{T}\right)\left(c^{(3)}-c^{(4)}\right), \\
s^{(3)}=-\left(s^{(1)}+s^{(2)}+s^{(4)}\right) .
\end{gathered}
$$

Figure 4.2 shows a schematic diagram of the geometry, and Tables 4.1, 4.2, and 4.3 contain geometric information, boundary conditions, and material parameters used in the simulation. Note that a very large value is selected for the stiffness parameter: $\gamma=10^{5}$.

Table 4.1. Geometric information for three-dimensional backward-facing step simulation.

\begin{tabular}{|l|l|}
\hline \multicolumn{1}{|c|}{ Quantity } & \multicolumn{1}{c|}{ Value } \\
\hline \hline Channel length upstream & 4 , from $x=-4$ to $x=0$ \\
\hline Channel length downstream & 6 , from $x=0$ to $x=6$ \\
\hline Step height & 0.5, from $y=-0.5$ to $y=0$ \\
\hline Channel height upstream & 0.5, from $y=0$ to $y=0.5$ \\
\hline Channel height downstream & 1, from $y=-0.5$ to $y=0.5$ \\
\hline Channel width & 1, from $z=0$ to $z=1$ \\
\hline Heated region & $-2 \leq x \leq 0, y=0,0 \leq z \leq 1$ \\
\hline Center line (plotting) & $-4 \leq x \leq 6, y=0, z=0.5$ \\
\hline
\end{tabular}

Table 4.2. Boundary conditions for three-dimensional backward-facing step simulation.

\begin{tabular}{|l|c|}
\hline \multicolumn{1}{|c|}{ Quantity } & Value \\
\hline \hline Velocity at inlet, reactive surface, other walls & $(1,0,0),(0,0,0),(0,0,0)$ \\
\hline Temperature at inlet, reactive surface, other walls & $1,5,1$ \\
\hline Species $1,2,4$ concentrations at inlet & $0.5,0.5,0$ \\
\hline Species $1,2,4$ fluxes at reactive surface, other walls & chemistry, 0 \\
\hline Velocity, temperature, species at outlet & natural boundary conditions \\
\hline
\end{tabular}


Table 4.3. Material parameters for three-dimensional backward-facing_step simulation.

\begin{tabular}{|l|c|}
\hline \multicolumn{1}{|c|}{ Quantity } & Value \\
\hline \hline Density & 1 \\
\hline Viscosity & 0.01 \\
\hline Specific heat & 1 \\
\hline Thermal conductivity & $1 / 71$ \\
\hline Species $1,2,4$ capacities & 1 \\
\hline Species $1,2,4$, diffusivities & $1 / 71$ \\
\hline Activation energy $E_{1}$ & 10 \\
\hline Activation energy $E_{2}$ & 1 \\
\hline Stiffness parameter $\gamma$ & $10^{5}$ \\
\hline
\end{tabular}

The mesh used for this problem is also shown in Figure 4.2. It is composed of 3700 27-node brick elements, yielding a total of about 20,000 nodes in the mesh and about 60,000 unknown concentrations (a modest three-dimensional problem). Nevertheless, the direct solver discussed in the previous chapter would require approximately 1.2 Gbyte of core memory for application to this problem. Thus, a segregated iterative approach is the only viable approach for large threedimensional multi-species problems.

Three simulations are performed for this problem. The original segregated solver and the stiff segregated solver are both applied to the full three-dimensional geometry in Figure 4.2, and the direct solver using the full-Jacobian approach previously discussed is applied to the two-dimensional geometry corresponding to the symmetry plane at $z=0.5$ of the full three-dimensional geometry. For all three simulations, there are two computational steps in the solution procedure. The original segregated solver in FIDAP is first applied to solve the velocity/pressure/temperature problem alone. The resulting velocity and temperature fields are then used as input for the subsequent solution of the species concentration fields. When using the segregated solvers, iterations are continued until the norm of the relative change of the solution falls below $10^{-4}$. Table 4.4 shows the computational times employed by the original and stiff segregated solvers following this procedure, where simulations are performed on a single node of an IBM RS6000.

Table 4.4. Simulation information for the original and stiff segregated solvers.

\begin{tabular}{|l|c|c|c|c|}
\hline \multicolumn{1}{|c|}{ Solver } & Iterations & Wall-Clock Time (h) & Time per Iter. (s) & Accuracy \\
\hline \hline Original segregated & 265 & 17 & 3.9 & Poor \\
\hline Stiff segregated & 164 & 15 & 5.8 & Good \\
\hline
\end{tabular}

The accuracy of the results produced by the original and stiff segregated solvers can be ascertained by comparing them along the symmetry plane to the fully converged results from the direct solver for the corresponding two-dimensional problem. Figure 4.3 shows the concentration profiles for species 1 and $4\left(c^{(2)}=1-c^{(1)}-c^{(4)}\right)$ calculated by all three methods along the center line in the symmetry 
plane (see Figure 4.2). Each species profile calculated by the stiff segregated solver is almost identical to the corresponding two-dimensional profile, whereas the profiles calculated by the original segregated solver differ greatly from the profiles calculated by the other two solvers. Note that the profiles from the original segregated solver differ substantially along the heated region from $1 / 3$, the large $\gamma$ limit of all species concentrations on the heated region for the prescribed surface chemistry (recall as previously discussed, the "concentration" of surface species 3 is not to be interpreted as a mass fraction). Thus, the stiff segregated solver has produced an accurate solution, whereas the original segregated solver has not produced an accurate solution.

The above observations indicate that the rate of approach to the converged solution by the original segregated solver has become extremely small even though the difference between the calculated and converged solutions is still large. This is borne out by examining the convergence behavior of both segregated solvers, as shown in Figure 4.4. The convergence rate with the original segregated solver becomes small quite rapidly, even though the solution is far away from the converged result. However, with the stiff segregated solver, the convergence rate plateaus at a higher value until the converged solution is closely approached. Figure 4.5 shows the concentration profiles with the stiff segregation solver after certain numbers of iterations. After only 31 iterations, the solution is already substantially better than that of the original segregated solver (see Figure 4.3). From 31 to 71 iterations, which corresponds roughly to the plateau in the convergence rate shown in Figure 4.4, further improvement occurs in the solution near and downstream of the heated region. By 101 iterations, the solution is almost indistinguishable from the converged result at 164 iterations. Thus, the stiff segregated solver converges faster to a more accurate solution than does the original solver.

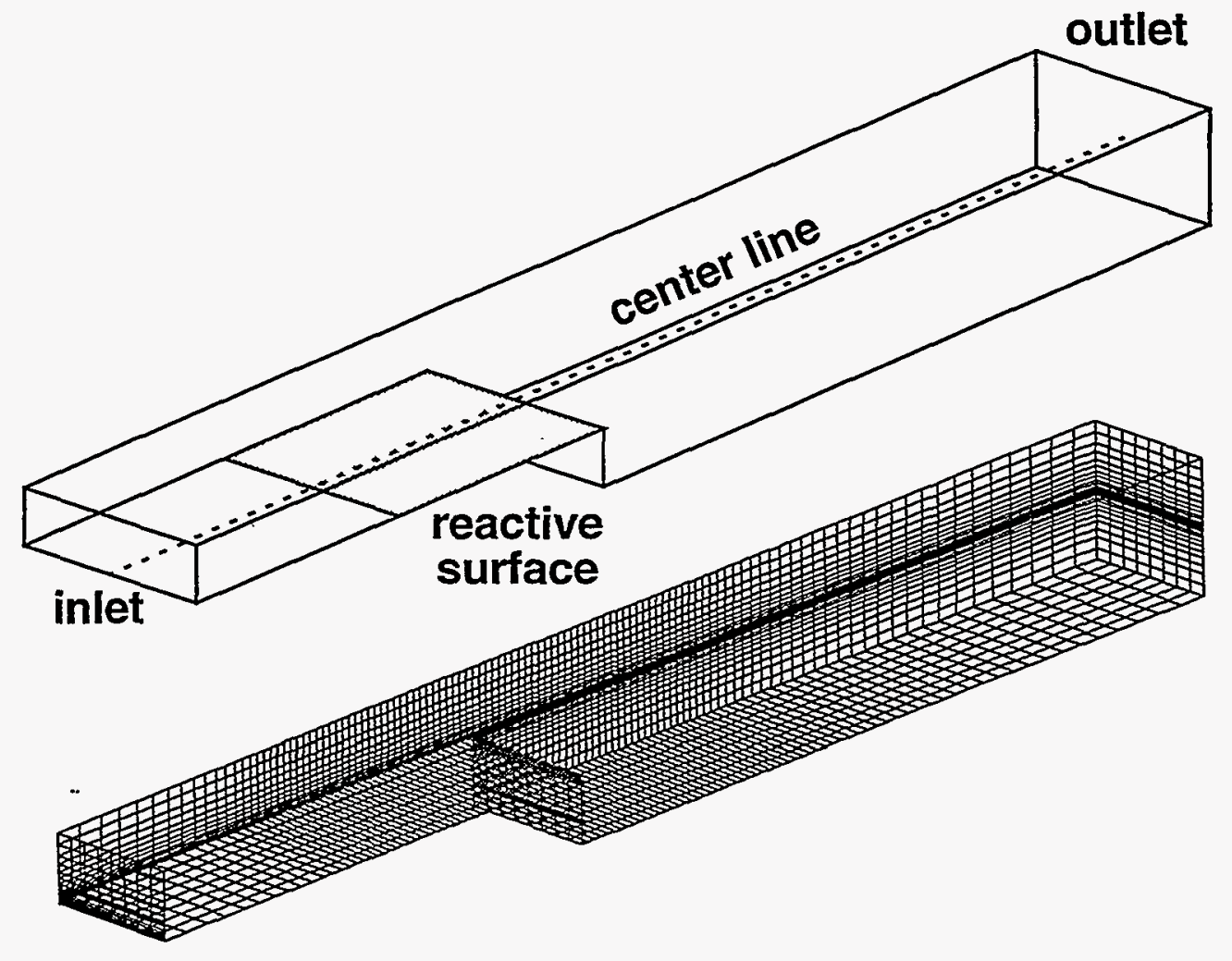

Figure 4.2. Three-dimensional geometry: top, schematic diagram; bottom, mesh. 


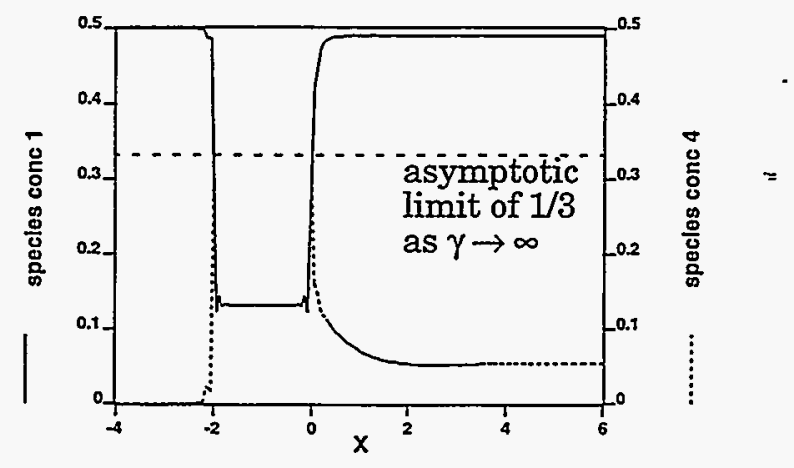

original segregated solver for 3D: 265 iterations

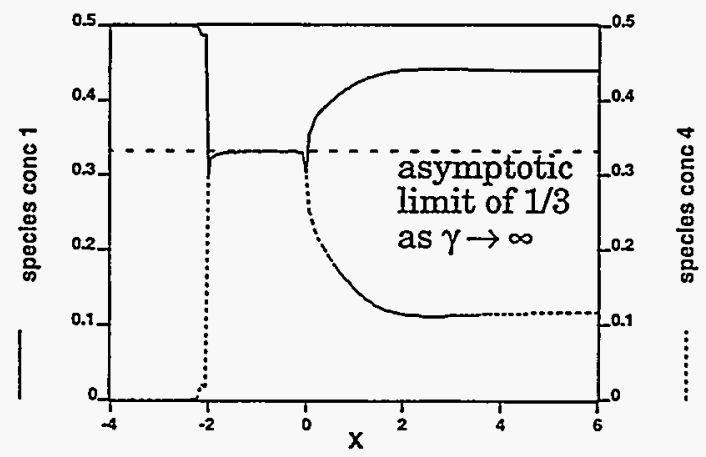

stiff segregated solver for 3D: 164 iterations

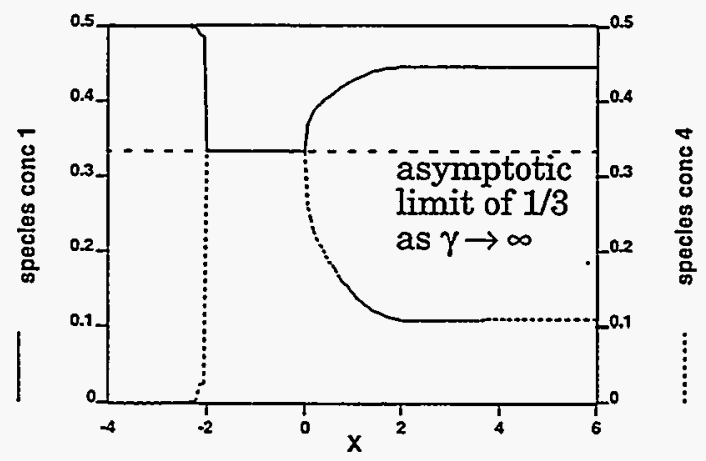

direct solver for corresponding 2D: converged

Figure 4.3. Solver dependence of solution along center line across reactive surface for $\gamma=10^{5}$. 

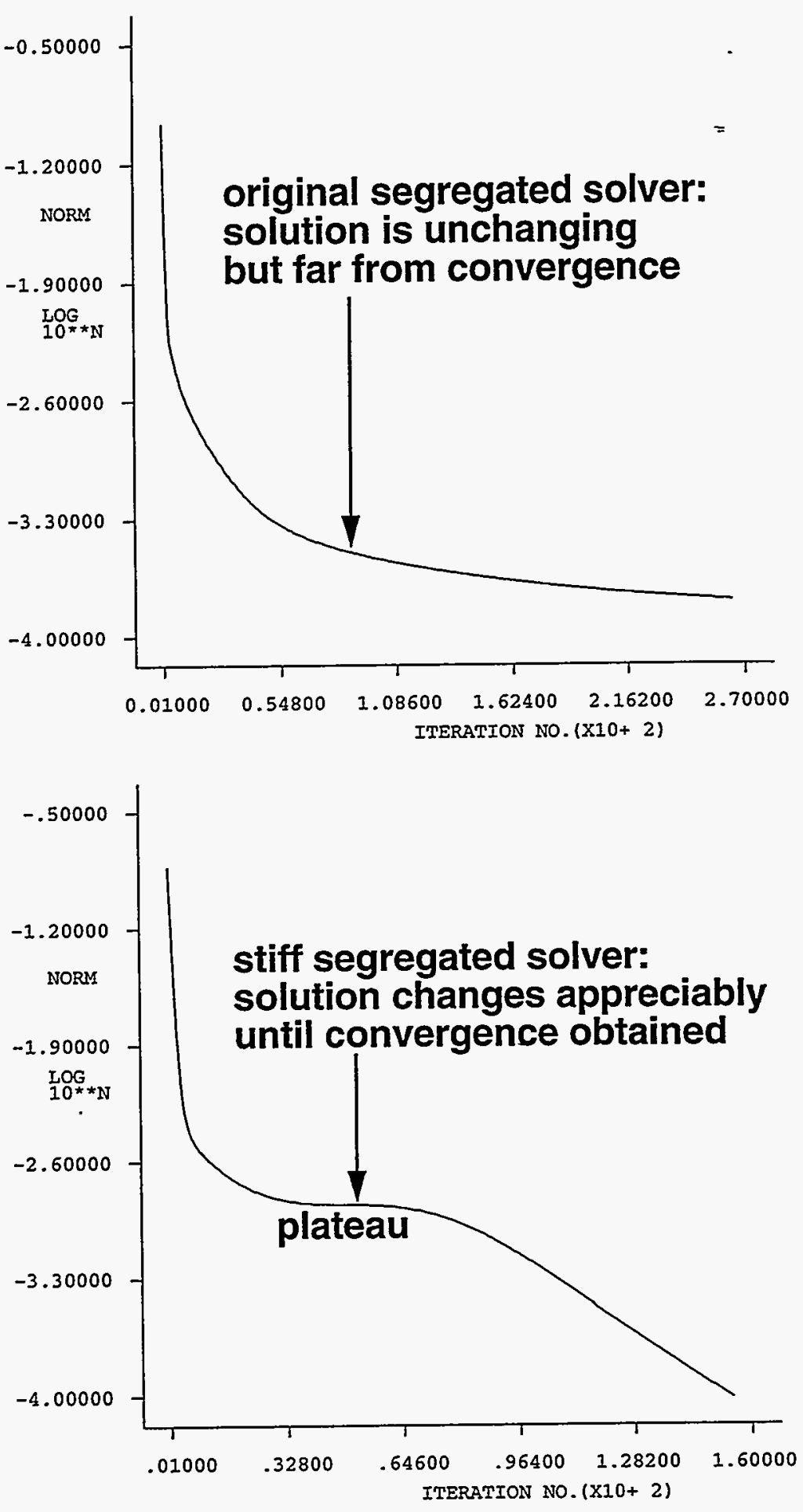

Figure 4.4. Convergence behavior of original and stiff segregated solver for $\gamma=10^{5}$. 


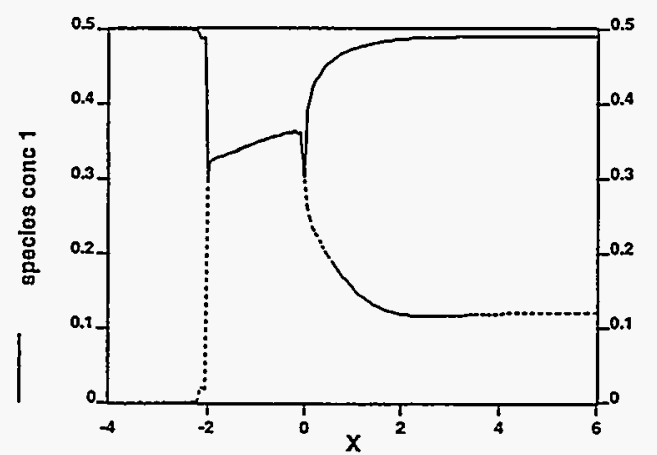

31 iterations

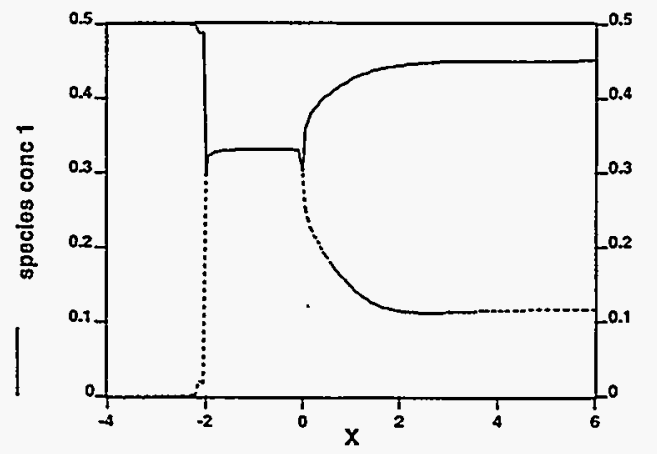

101 iterations

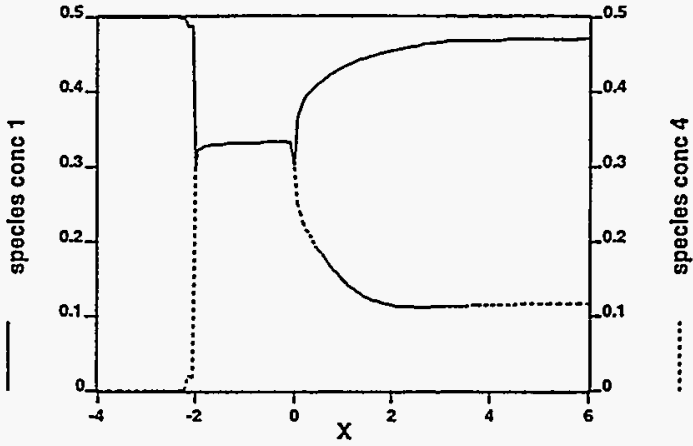

71 iterations

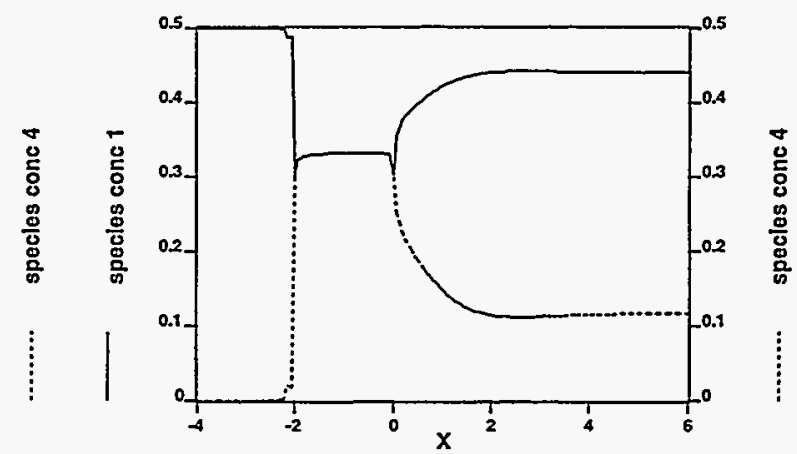

164 iterations (converged)

Figure 4.5. Center-line solution behavior of stiff segregated solver prior to convergence. 


\section{Conclusions}

In support of the Motorola CRADA, the computational fluid dynamics code FIDAP (Fluid Dynamics International) has been examined for simulating semiconductor-processing applications. These applications typically require the solution of flow/thermal/chemistry problems with three-dimensional geometries and multi-species mixtures, including species that exist only on rēactive surfaces. FIDAP's capabilities have been assessed for problems with these characteristics, with particular emphasis on treating surface species, stiff chemical reactions (rapid reaction rates compared with transport rates), and large problems (three-dimensional and/or multi-species). Where needed, FIDAP's capabilities have been enhanced within the constraints of the overall FIDAP architecture. A method for treating surface species in FIDAP has been demonstrated. The method requires no modifications to the FIDAP source code or custom subroutines but instead relies on a novel application of FIDAP commands. For problems with stiff chemistry, a coupled algorithm utilizing the full Jacobian for the chemical-reaction source terms has been implemented in FIDAP. Good convergence behavior is obtained even on extremely stiff test problems. For extremely large problems resulting from three-dimensionality or many species, a stiff segregated algorithm has been developed and implemented in FIDAP. Test problems that are too large for the direct solver and too stiff for the original segregated solver are solved accurately by the stiff segregated solver. However, upwinding must be used to maintain algorithm stability for convection-dominated problems. These enhancements will facilitate solution of chemically reacting flow problems of the type found in semiconductor-processing applications. 


\section{References}

[1] Fluid Dynamics International, FIDAP Users Manual, version. 7.0 (Fluid Dynamics International, Evanston, IL, 1993).

[2] E. W. Egan, "Modeling Transport Phenomena at Motorola," presented at the Sixth FIDAP Users Conference, April 30-May 2, 1995, Chicago, IL.

[3] T. A. Baer and J. R. Torczynski, "Solving Stiff Chemistry Problems with FIDAP" in Proceedings of the Sixth FIDAP Users Conference, edited by C. Rosenblat (Fluid Dynamics International, Evanston, IL, 1995), Section 11. 


\section{Distribution}

\begin{tabular}{|c|c|c|c|}
\hline MS 0601 & 1126 & H. K. & Moffat \\
\hline MS 1078 & 1302 & C. W. & Gwyn \\
\hline MS 0603 & 1314 & P. & Esherick \\
\hline MS 9042 & 8303 & R. J. & Kee \\
\hline MS 9042 & 8345 & P. A. & Spence \\
\hline MS 0841 & 9100 & P. J. & Hommert \\
\hline MS 0828 & 9102 & R. D. & Skocypec \\
\hline MS 0833 & 9103 & J. $\mathrm{H}$. & Biffle \\
\hline MS 0828 & 9104 & E. D. & Gorham \\
\hline MS 0826 & 9111 & W. L. & Hermina \\
\hline MS 0826 & 9111 & C. E. & Hickox \\
\hline MS 0826 & 9111 & R. R. & Rao \\
\hline MS 0826 & 9111 & P. $R$. & Schunk \\
\hline MS 0834 & 9112 & A. C. & Ratzel \\
\hline MS 0834 & 9112 & T. A. & Baer (10) \\
\hline MS 0834 & 9112 & F. & Gelbard \\
\hline MS 0834 & 9112 & J. R. & Torczynski (10) \\
\hline MS 0835 & 9113 & T. C. & Bickel \\
\hline MS 0827 & 9114 & R. T. & McGrath \\
\hline MS 0827 & 9114 & J. E. & Brockmann \\
\hline MS 0827 & 9114 & A. $\mathrm{S}$. & Geller \\
\hline MS 0827 & 9114 & D. J: & Rader \\
\hline MS 0825 & 9115 & W. H. & Rutledge \\
\hline MS 0836 & 9116 & C. W. & Peterson \\
\hline MS 0443 & 9117 & H. S. & Morgan \\
\hline MS 0437 & 9118 & E. P. & Chen, actg. \\
\hline MS 9018 & $8523-2$ & Central & I Technical Files (1) \\
\hline MS 0899 & 4414 & Technic & cal Library (5) \\
\hline MS 0619 & 12630 & $\begin{array}{l}\text { Review } \\
\text { for DOI }\end{array}$ & $\begin{array}{l}\text { and Approval (2) } \\
\text { E/OSTI }\end{array}$ \\
\hline
\end{tabular}

Dr. Erik W. Egan

Motorola, Inc.

Semiconductor Products Sector 3501 Ed Bluestein Blvd., TX11

Mail Stop K10

Austin, TX 78721

Dr. Michael S. Engelman

Fluid Dynamics International

500 Davis Street, Suite 600

Evanston, IL 60201 
